\title{
Structure and function analysis of adiponectin receptors toward development of novel antidiabetic agents promoting healthy longevity
}

\author{
Miki Okada-Iwabu' ${ }^{1), 2)}$, Masato Iwabu'1),3),4), Toshimasa Yamauchi ${ }^{1), 5)}$ and Takashi Kadowaki1),6),7) \\ 1) Department of Diabetes and Metabolic Diseases, Graduate School of Medicine, The University of Tokyo, Tokyo, Japan \\ 2) Laboratory for Advanced Research on Pathophysiology of Metabolic Diseases, The University of Tokyo, Tokyo, Japan \\ 3) Department of Integrated Molecular Science on Metabolic Diseases, 22nd Century Medical and Research Center, The University of \\ Tokyo, Tokyo, Japan \\ 4) PRESTO, Japan Science and Technology Agency, Kawaguchi, Saitama, Japan \\ 5) AMED-CREST, Japan Agency for Medical Research and Development, Tokyo, Japan \\ 6) Department of Prevention of Diabetes and Life-style Related Diseases, The University of Tokyo, Tokyo, Japan \\ 7) Department of Metabolism and Nutrition, Teikyo University Mizonokuchi Hospital, Kawasaki, Kanagawa, Japan
}

\begin{abstract}
Plasma adiponectin levels and expression of its receptors, AdipoRs are decreased in obesity, which cause insulin resistance and diabetes. AdipoR-deficient mice exhibit insulin resistance and impaired glucose tolerance. Moreover, newly identified AdipoR agonists not only improve insulin resistance but prolong lifespan shortened by obesity via AdipoR. Furthermore, efforts to promote structure-based drug discovery research at our laboratory have led to the first ever successful crystallization of AdipoR as well as to clarification of their structures. Structural analysis of AdipoRs as key molecules in lifestyle-related diseases is thus expected to lead not only to the acceleration of structure-based drug discovery but to the elucidation of novel aspects of the AdipoR structures and functions in the years to come. Finally, with the development of novel AdipoR-targeted antidiabetic agents also capable of prolonging lifespan, the attainment of healthy longevity may finally be brought within reach.
\end{abstract}

Key words: Adiponectin, AdipoR, Structure, Diabetes, Obesity

\section{Introduction}

The number of patients with diabetes continues to increase drastically worldwide. According to the report of the International Diabetes Federation (IDF), the number of those with diabetes in the world stands at 425 million, as of 2017, and is expected to rise to 693 million by 2045; likewise, obesity is increasing worldwide, with the number of overweight adults and children estimated at 2.2 billion, as of 2015 , which accounts for about $30 \%$ of the world's entire population [1]. Against a background

Submitted Aug. 1, 2018; Accepted Sep. 18, 2018 as EJ18-0310 Released online in J-STAGE as advance publication Oct. 3, 2018 Correspondence to: Miki Okada-Iwabu, Department of Diabetes and Metabolic Diseases, Graduate School of Medicine, The University of Tokyo, 7-3-1 Hongo, Bunkyo-ku, Tokyo 113-0033, Japan.

E-mail: omiki-tky@umin.ac.jp of insulin resistance, obesity is shown to induce diabetes, dyslipidemia and hypertension or the so-called metabolic syndrome (MS), thus accounting for a high incidence of cardiovascular disease in affected individuals $[2,3]$. Given that individuals with the metabolic syndrome are thought to be at high risk of developing diabetes, it is critically important that the etiology of obesity, insulin resistance, diabetes and their complications be unraveled, so that resulting insights may be used to establish radical preventive and therapeutic measures for these conditions and diseases.

Adiponectin [4-7], a physiologically active substance secreted from adipocytes, is decreased with obesity, and systemically decreased secretion of adiponectin and decreased action of its receptors (AdipoR1 and AdipoR2; AdipoRs) [8] are primarily responsible for worldwide explosion of lifestyle-related diseases. Adiponectin possesses insulin-sensitizing properties, and insulin resist- 
ance and impaired glucose tolerance are shown to occur in AdipoR-deficient mice [9-11]. Furthermore, adiponectin and AdipoR1 are shown to improve mitochondrial function in skeletal muscle, thereby improving glucose/ lipid metabolism [12]. Following these observations, we went on to identify small-molecule compounds that become activated by binding to the AdipoRs and demonstrated that they improve diabetes and prolong lifespan cut short by obesity, thus paving the way for their clinical development [13]. In pursuit of AdipoR structurebased drug discovery research, we have successfully crystalized AdipoRs, presumably the least amenable of all transmembrane proteins to crystallization, and clarified their structures for the first time [14, 15]. AdipoRs represent a novel class of transmembrane proteins whose topology is opposite to that of G protein-coupled receptors (GPCRs) and whose structures incorporate a zinc ion and are distinct from those of GPCRs.

Seeing that controlling AdipoR-mediated signaling may have potential as therapeutic modalities for obesity and type 2 diabetes, we identified AdipoRon, an AdipoR agonist, and have since unremittingly pursued structurebased drug discovery research. In this article, we provide an overview of AdipoR-targeted structural analysis and describe future expectations and prospects for the treatment of obesity and type 2 diabetes in this context [16, 17].

\section{Protein Structure of Adiponectin}

Adiponectin is a $30-\mathrm{kDa}$ glycoprotein and is composed of an N-terminal signal sequence, a nonhomologous or hypervariable region, a collagen-like sequence and a C-terminal globular region [18, 19]. Adiponectin multimeric complexes have been identified as low molecular weight form (LMW, trimer, $\sim 90 \mathrm{kDa}$ ), medium molecular weight form (MMW, hexamer, $\sim 180 \mathrm{kDa}$ ), and high molecular form (HMW, 12-18 monomers, $\sim 360-540 \mathrm{kDa}$ ) [20]. Multimeric complexes contribute significantly to the numerous biological effects of adiponectin, with its multimer formation dependent on the formation of disulfide bonds mediated by Cys-39 [21]. With regard to the crystal structure of adiponectin, only reported that the structure of a single-chain trimer of the human adiponectin globular domain has been clarified [22]. Thus, the structural information about adiponectin multimeric complexes, if available, should prove critically important for the understanding the adiponectin signaling mechanism.

\section{Identification of AdipoRs}

In obesity as a disease, decreased secretion of adiponectin is primarily responsible for the onset of diabetes, the MS and associated macroangiopathy not only due to its associated risk factors for diabetes and dyslipidemia but also through its direct effects on vascular walls [23-26]. In this context, our research efforts focused on identifying AdipoRs, particularly given that adiponectin action-activating therapeutic modalities may constitute a radical therapeutic approach to diabetes and macroangiopathy and that unraveling the mechanisms of action of adiponectin may provide important clues as to the radical treatment of diabetes and MS.

AdipoR1 and AdipoR2 were identified as adiponectin receptors in 2003 [8]. AdipoR1 was found to be expressed relatively ubiquitously as well as abundantly in the skeletal muscle and the liver, while AdipoR2 was found to be expressed particularly abundantly in the liver. The hallmark features of AdipoR1/AdipoR2 are that their topology is opposite to that of all GPCRs reported to date, with their $\mathrm{N}$ - and $\mathrm{C}$-terminus predicted to be intracellularly and extracellularly located, respectively. In the studies that followed, we went on to genetically engineer AdipoR1- and AdipoR2-deficient mice and found that AdipoR1/AdipoR2 double knockout mice were associated with loss of adiponectin binding and action, thus providing evidence that AdipoR1/AdipoR2 represent major AdipoRs at work in the body [11]. AdipoR1/AdipoR2 double knockout mice were associated with insulin resistance and impaired glucose tolerance, demonstrating that the mechanisms involved include increased inflammation and oxidative stress in organs essential to metabolism (e.g., liver, skeletal muscle and adipose tissue), increased gluconeogenesis, and decreased glucose uptake [11].

\section{Physiological and Pathophysiological Role of AdipoR1/AdipoR2}

AdipoR expression is decreased in animal models of obesity/type 2 diabetes and decreased AdipoR expression is causally implicated in obesity-linked diseases [11]. Of note, in the liver, AdipoR1 was found to activate the AMPK pathway, while AdipoR2 was found to activate PPAR $\alpha$, promote fatty acid oxidation/energy expenditure, and exert anti-inflammatory/anti-oxidative stress effects, thereby improving glucose tolerance [11].

Furthermore, in skeletal muscle, adiponectin/AdipoR1 
signaling increases mitochondrial volume and function thereby improving glucose/lipid metabolism and enhancing endurance [12]. PPAR $\gamma$ coactivator- $1 \alpha$ (PGC-1 $\alpha$ ), mitochondrial volume/function, and type 1 fibers are decreased, leading to decreased endurance as well as insulin resistance in skeletal muscle-specific AdipoR1deficient mice [12]. Furthermore, via AdipoR1, adiponectin not only increases intracellular calcium concentrations but activates the AMPK pathway/the longevity gene SIRT1, thus accounting for exercise-mimicking signaling [12].

\section{Other Candidate Receptors and Pathways}

It has been reported that T-cadherin is a receptor for hexameric and high-molecular-weight forms of Acrp30/ adiponectin [27] but T-cadherin has no transmembrane or intracellular domain required for adiponectin signaling. This is supported by studies showing failure of adiponectin to bind to cardiac tissue in T-cadherin-deficient mice [28].

T-cadherin bind to HMW as well as hexameric forms of adiponectin, but not to the trimeric, globular, or bacterially produced multimers of adiponectin [27]. In Tcadherin-deficient mice, circulating levels of adiponectin, particularly HMW forms of adiponectin, are elevated [28]. In addition, adiponectin levels are dependent on T-cadherin and regulate tissue T-cadherin levels by suppressing GPI phospholipase-mediated release of Tcadherin from the cell surface [29]. T-cadherin is essential for adiponectin-mediated revascularization after chronic ischemia by promoting cell migration and proliferation [30]. Interestingly, T-cadherin is critical for adiponectin-mediated cardioprotection in mice [28].

Adiponectin has also been reported to modulate inflammatory reactions via calreticulin, which along with CD91, is involved in the adiponectin-mediated uptake of apoptotic cells [31]. Adiponectin has also been shown to be capable of opsonizing apoptotic cells, and phagocytosis of cell corpses is shown to be mediated by the binding of adiponectin to calreticulin on the macrophage cell surface [31].

\section{Discovery of AdipoR Agonist}

The metabolic environment of an individual organism may be made amenable to normalization through activation of adiponectin/AdipoR signaling thereby causing qualitative changes in its metabolic capacity. Indeed, adi-
ponectin/AdipoR-activating agents appear to have potential as "exercise-mimicking agents" and are expected to open up a new avenue in the development of radical treatments for MS, type 2 diabetes, and atherosclerosis. Therefore, they appear to hold great promise as highly effective therapeutic agents for individuals who have difficulties exercising due to their medical conditions or locomotor disorders.

Drawing on such resources as the Compound Library available at the Drug Discovery Open Innovation Center (currently, Drug Discovery Initiative; DDI), the University of Tokyo, we screened candidate compounds and succeeded in identifying a prototype small-molecule AdipoR-activating compound (Adiponectin Receptor agonist; AdipoRon) [13]. AdipoRon is shown to activate the AMPK pathway and enhance mitochondrial function, as well as exert antidiabetic properties via AdipoR [13].

Surprisingly, it has become clear that AdipoR1deficient mice and AdipoR2-deficient mice have a shorter lifespan than wild-type mice, with the AdipoR1/R2 double knockout mice found to have the shortest lifespan [13]. In contrast, it was shown that the use of AdipoRon leads to restoration of lifespan cut short by obesity in mouse models of obesity/type 2 diabetes despite their being fed a high-fat diet, suggesting that AdipoR signaling is associated with lifespan prolongation [13]. Discovery of candidate small-molecule AdipoR-activating compounds and its development of AdipoR-activating agents have been expected to provide novel and effective treatments for the obesity-associated diseases, which contribute to healthy longevity (Fig. 1).

\section{Structural Analysis of AdipoR1/AdipoR2}

At the time of the discovery of AdipoR1/AdipoR2, they were assumed to be seven transmembrane proteins whose topology was predicted to be opposite to that of GPCRs. Of note, while the mechanisms of activation of GPCRs began to be unraveled though structural analysis of GPCR/G-protein complexes, the AdipoR1/AdipoR2 structures remained largely unknown. Thus, our research efforts focused on elucidating the AdipoR1/AdipoR2 structures as well as their functions, as we went forward with structure-based drug discovery research.

During the course of this research, an AdipoR1/ AdipoR2 expression and purification system was constructed [14], and an antibody was engineered for AdipoR1/AdipoR2 structure recognition. Then, AdipoR1/ AdipoR2/antibody complexes were crystalized in a lipid 


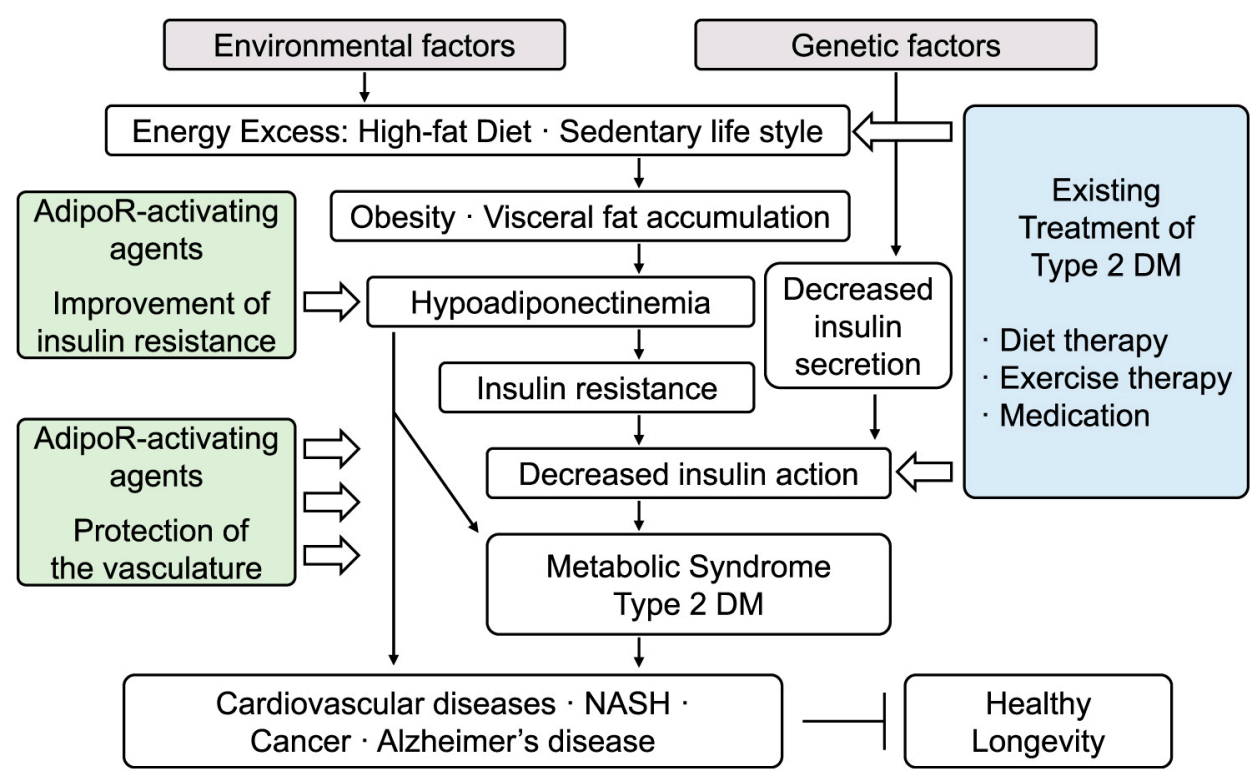

Fig. 1 Potential strategies for AdipoR-activating agents in the treatment of obesity-linked diseases. Decreased adiponectin effects are causally implicated in obesity-linked diseases, such as type 2 DM (diabetes mellitus), cardiovascular diseases and cancer. Increased activation of AdipoR pathways improve insulin resistance and adiponectin has protective vascular effects. The drugs targeted at AdipoR pathways may have beneficial effects on healthy longevity and obesity-linked diseases.

mesophase, and, of all crystals thus obtained, the crystal structures of AdipoR1 and AdipoR2 were determined at resolution values of $2.9 \AA$ and $2.4 \AA$, respectively [15].

AdipoR1 and AdipoR2 were each shown to consist of an N-terminal intracellular region, a short intracellular helix, 7 transmembrane helices, and a C-terminal extracellular region, with the $\mathrm{N}$-terminal intracellular region shown to have been recognized by the antibody used in crystalizing AdipoR1 and AdipoR2, which were found to be similar in structure [15].

With its C-terminus located extracellularly, the topology of the seven transmembrane domain of AdipoR1/ AdipoR2 relative to the plasma membrane was opposite to that of conventional seven transmembrane proteins, such as GPCRs [15]. It was also shown that the structural features of GPCRs, such as proline-induced kinks of the transmembrane helices, are not seen in those of AdipoR1/AdipoR2; instead, the transmembrane helices of AdipoR1/AdipoR2 are not kinked, with helix V shown to be slightly curved due to the presence of 3 Gly residues [15]. It was thus concluded that AdipoR1 and AdipoR2 possess novel structures.

\section{The Zinc-Binding Sites and Internal Cavities of AdipoR1/AdipoR2}

The AdipoR1/AdipoR2 structures are each shown to have a zinc ion bound within their seven transmembrane domain, with the zinc-binding sites located at a distance of about $4 \AA$ from the inner surface of the plasma membrane and with the zinc ion coordinated by 3 His residues [15]. Remarkably, a water molecule was shown to be in place between the zinc ion and the Asp side-chain carboxylic group in AdipoR2, with the zinc ion binding helices II, III, and VII together, thus likely contributing to stabilization of the subdomain structure consisting of helices I, II, III and VI [15]. It was also shown that these 3 His/Asp residues are conserved in the homologues of AdipoR1 and AdipoR2 [15].

Furthermore, an examination of the activity of AdipoR1/AdipoR2 through substitution by Ala of the zinc ion-coordinating amino acid residues suggested that the zinc ion bound within AdipoR1 may not be directly implicated in AMPK activation but may contribute to the maintenance of the AdipoR1 structure, while that bound within AdipoR2 may not only contribute to the maintenance of the AdipoR2 structure but may also affect AdipoR2 signaling [15].

In addition, an internal cavity, which includes a zinc- 
binding site, was shown to be present, surrounded by the seven transmembrane domain, in the AdipoR1 and AdipoR2 structures [15]. It was also shown that this cavity consists of two subdomains extending from the cytoplasmic surface to the middle of the outer lipid layer of the membrane, and contains unidentified electron densities [15].

\section{Extracellular Surfaces of AdipoR1 and AdipoR2}

Research has also shown that the extracellular surfaces of AdipoR1/AdipoR2 each consist of 3 extracellular loops and a C-terminal extracellular region, with the $\mathrm{C}$ terminal 2 turns of transmembrane helix VII protruding from the extracellular surface and the part of the Cterminal region that follows helix VII appearing to be highly flexible and unstructured [15].

An earlier experiment with a yeast two-hybrid cDNA library demonstrated that adiponectin interacts with the C-terminal extracellular region of AdipoR1/AdipoR2 [32]. In this context, AdipoR-mediated AMPK activation is not affected in AdipoR1 mutants with deletion of the C-terminal region that follows helix VII but is decreased in AdipoR1 mutants with deletion of the C-terminal region including the 2 transmembrane helix VII amino acid residues, suggesting that the $\mathrm{C}$-terminal 2 turns of helix VII protruding from the extracellular surface may be implicated in adiponectin signaling [15].

Examination of AdipoR1/AdipoR2 activity at our laboratory through substitution of the conserved extracellular loop amino acid residues with Gly/Ser and demonstrated that AdipoR-mediated AMPK activation is decreased in AdipoR1 mutants with substitution of all 3 extracellular loop amino acid residues by Gly/Ser and deletion of the $\mathrm{C}$-terminal region including the 2 helix VII amino acid residues, which suggested that AdipoR1 may interact with adiponectin on a broad extracellular surface involving the 3 extracellular loops and the Cterminal region that follows helix VII [15].

AdipoR recognizes adiponectin by the extensive use of its extracellular face [15]. Therefore, it remains extremely important to clarify of the crystal structure of adiponectin-AdipoR complex.

\section{Structure and Function Analysis of AdipoR1/AdipoR2}

AdipoR1 and AdipoR2, are shown to be completely different in structure from GPCRs [15]. Indeed, our research has revealed that they have novel structures, each containing an internal cavity inside the 7 transmembrane helices coordinating a zinc ion, demonstrating that their structures and functions are novel and distinct from those of GPCRs [15].

A recent report by Vasiliauskaité-Brooks et al. [33] has provided further insights into the AdipoR1/AdipoR2 structures, demonstrating that AdipoR1/AdipoR2 have enzymatic activity as their novel function, with the caveat, however, that they exhibit extremely low ceramidase activity, thus requiring further research to characterize the enzymatic parameters for their activity as well as their substrate specificity. Therefore, further research insights are eagerly awaited into the AdipoR1/AdipoR2 structures and their substrate specificity for their enzymatic activity, as well as their additional yet unidentified functions.

\section{Conclusion}

In summary, structural analysis of AdipoR1/AdipoR2 has revealed that AdipoR1/AdipoR2 possess structures and functions that are completely different from those of GPCRs, thus establishing AdipoR1/AdipoR2 as a new class of receptors. Thus, structural analysis of AdipoR1/ AdipoR2 is expected to lead not only to elucidation of as yet unidentified AdipoR signaling but to development of small-molecule AdipoR-activating compounds, such as AdipoRon, and their optimization for human use. Finally, as research proceeds from discovery of candidate smallmolecule AdipoR-activating compounds to development of AdipoR-activating medicines, it is expected to lead to the development of novel and effective treatments for the metabolic syndrome and diabetes, thus finally bringing within reach the attainment of healthy longevity.

\section{Acknowledgements}

We thank N. Ohuchi and K. Miyata for help in preparing the manuscript. This work was supported by JSPS KAKENHI Grant Numbers JP16K15487, JP26000012, JP26293216, 18K10988, and by JST, PRESTO (JPMJPR13MF).

\section{Disclosure}

None of the authors have any potential conflicts of interest associated with this research. 


\section{References}

1. Collaborators GBDO, Afshin A, Forouzanfar $\mathrm{MH}$, Reitsma MB, Sur P, et al. (2017) Health effects of overweight and obesity in 195 countries over 25 years. $N$ Engl J Med 377: 13-27.

2. Matsuzawa Y (1997) Pathophysiology and molecular mechanisms of visceral fat syndrome: the Japanese experience. Diabetes Metab Rev 13: 3-13.

3. Reaven G (2012) Insulin resistance and coronary heart disease in nondiabetic individuals. Arterioscler Thromb Vasc Biol 32: 1754-1759.

4. Scherer PE, Williams S, Fogliano M, Baldini G, Lodish HF (1995) A novel serum protein similar to C1q, produced exclusively in adipocytes. J Biol Chem 270: 2674626749.

5. Hu E, Liang P, Spiegelman BM (1996) AdipoQ is a novel adipose-specific gene dysregulated in obesity. $\mathrm{J} \mathrm{Biol} \mathrm{Chem}$ 271: 10697-10703.

6. Maeda K, Okubo K, Shimomura I, Funahashi T, Matsuzawa Y, et al. (1996) cDNA cloning and expression of a novel adipose specific collagen-like factor, apM1 (AdiPose Most abundant Gene transcript 1). Biochem Biophys Res Commun 221: 286-289.

7. Nakano Y, Tobe T, Choi-Miura NH, Mazda T, Tomita M (1996) Isolation and characterization of GBP28, a novel gelatin-binding protein purified from human plasma. $J$ Biochem 120: 803-812.

8. Yamauchi T, Kamon J, Ito Y, Tsuchida A, Yokomizo T, et al. (2003) Cloning of adiponectin receptors that mediate antidiabetic metabolic effects. Nature 423: 762-769.

9. Yamauchi T, Kamon J, Waki H, Terauchi Y, Kubota N, et al. (2001) The fat-derived hormone adiponectin reverses insulin resistance associated with both lipoatrophy and obesity. Nat Med 7: 941-946.

10. Yamauchi T, Kamon J, Minokoshi Y, Ito Y, Waki H, et al. (2002) Adiponectin stimulates glucose utilization and fatty-acid oxidation by activating AMP-activated protein kinase. Nat Med 8: 1288-1295.

11. Yamauchi T, Nio Y, Maki T, Kobayashi M, Takazawa T, et al. (2007) Targeted disruption of AdipoR1 and AdipoR2 causes abrogation of adiponectin binding and metabolic actions. Nat Med 13: 332-339.

12. Iwabu M, Yamauchi $T$, Okada-Iwabu $M$, Sato $K$, Nakagawa T, et al. (2010) Adiponectin and AdipoR1 regulate PGC-1alpha and mitochondria by $\mathrm{Ca}(2+)$ and AMPK/SIRT1. Nature 464: 1313-1319.

13. Okada-Iwabu $M$, Yamauchi $T$, Iwabu $M$, Honma $T$, Hamagami K, et al. (2013) A small-molecule AdipoR agonist for type 2 diabetes and short life in obesity. Nature 503: 493-499.

14. Tanabe H, Motoyama K, Ikeda M, Wakiyama M, Terada $\mathrm{T}$, et al. (2015) Expression, purification, crystallization, and preliminary X-ray crystallographic studies of the human adiponectin receptors, AdipoR1 and AdipoR2. $J$ Struct Funct Genomics 16: 11-23.

15. Tanabe H, Fujii Y, Okada-Iwabu M, Iwabu M, Nakamura $\mathrm{Y}$, et al. (2015) Crystal structures of the human adiponectin receptors. Nature 520: 312-316.

16. Okada-Iwabu $M$, Iwabu $M$, Ueki $K$, Yamauchi $T$, Kadowaki T (2015) Perspective of small-molecule AdipoR agonist for type 2 diabetes and short life in obesity. Diabetes Metab J 39: 363-372.

17. Iwabu M, Okada-Iwabu M, Yamauchi T, Kadowaki T (2015) Adiponectin/adiponectin receptor in disease and aging. NPJ Aging Mech Dis 1: 15013.

18. Fruebis J, Tsao TS, Javorschi S, Ebbets-Reed D, Erickson MR, et al. (2001) Proteolytic cleavage product of 30-kDa adipocyte complement-related protein increases fatty acid oxidation in muscle and causes weight loss in mice. Proc Natl Acad Sci U S A 98: 2005-2010.

19. Berg AH, Combs TP, Scherer PE (2002) ACRP30/ adiponectin: an adipokine regulating glucose and lipid metabolism. Trends Endocrinol Metab 13: 84-89.

20. Fang H, Judd RL (2018) Adiponectin regulation and function. Compr Physiol 8: 1031-1063.

21. Pajvani UB, Du X, Combs TP, Berg AH, Rajala MW, et al. (2003) Structure-function studies of the adipocytesecreted hormone Acrp30/adiponectin. Implications fpr metabolic regulation and bioactivity. $J$ Biol Chem 278: 9073-9085.

22. Min X, Lemon B, Tang J, Liu Q, Zhang R, et al. (2012) Crystal structure of a single-chain trimer of human adiponectin globular domain. FEBS Lett 586: 912-917.

23. Arita Y, Kihara S, Ouchi N, Takahashi M, Maeda K, et al. (1999) Paradoxical decrease of an adipose-specific protein, adiponectin, in obesity. Biochem Biophys Res Commun 257: 79-83.

24. Li S, Shin HJ, Ding EL, van Dam RM (2009) Adiponectin levels and risk of type 2 diabetes: a systematic review and meta-analysis. JAMA 302: 179-188.

25. Lindsay RS, Funahashi T, Hanson RL, Matsuzawa Y, Tanaka S, et al. (2002) Adiponectin and development of type 2 diabetes in the Pima Indian population. Lancet 360 : $57-58$.

26. Kumada M, Kihara S, Sumitsuji S, Kawamoto $T$, Matsumoto S, et al. (2003) Association of hypoadiponectinemia with coronary artery disease in men. Arterioscler Thromb Vasc Biol 23: 85-89.

27. Hug C, Wang J, Ahmad NS, Bogan JS, Tsao TS, et al. (2004) T-cadherin is a receptor for hexameric and highmolecular-weight forms of Acrp30/adiponectin. Proc Natl Acad Sci U S A 101: 10308-10313.

28. Denzel MS, Scimia MC, Zumstein PM, Walsh K, Ruiz- 
Lozano P, et al. (2010) T-cadherin is critical for adiponectin-mediated cardioprotection in mice. J Clin Invest 120: 4342-4352.

29. Matsuda K, Fujishima Y, Maeda N, Mori T, Hirata A, et al. (2015) Positive feedback regulation between adiponectin and T-cadherin impacts adiponectin levels in tissue and plasma of male mice. Endocrinology 156: 934-946.

30. Parker-Duffen JL, Nakamura K, Silver M, Kikuchi R, Tigges U, et al. (2013) T-cadherin is essential for adiponectin-mediated revascularization. J Biol Chem 288: 24886-24897.

31. Takemura Y, Ouchi N, Shibata R, Aprahamian T, Kirber
MT, et al. (2007) Adiponectin modulates inflammatory reactions via calreticulin receptor-dependent clearance of early apoptotic bodies. J Clin Invest 117: 375-386.

32. Mao X, Kikani CK, Riojas RA, Langlais P, Wang L, et al. (2006) APPL1 binds to adiponectin receptors and mediates adiponectin signalling and function. Nat Cell Biol 8: 516-523.

33. Vasiliauskaite-Brooks I, Sounier R, Rochaix P, Bellot G, Fortier M, et al. (2017) Structural insights into adiponectin receptors suggest ceramidase activity. Nature 544: 120123. 